\title{
RETALHO PEDICULADO DO MÚSCULO GRANDE DORSAL PARA REPARO DE DEFEITO DIAFRAGMÁTICO EXPERIMENTAL EM CÃES
}

\section{LATISSIMUS DORSI MUSCULAR FLAP FOR REPAIR OF EXPERIMENTAL DIAPHRAGMATIC DEFECT IN DOGS}

\author{
Luciana Oliveira de Oliveira ${ }^{1}$ Ney Luis Pippi ${ }^{2}$ Dominguita Lühers Graça ${ }^{3}$ Renato Xavier Faria ${ }^{4}$ \\ Luciana Dambrósio Guimarães ${ }^{4}$ Alonso Gabriel Pereira Guedes ${ }^{4}$ Alexandre Mazzanti $^{4}$
}

RESUMO

Neste experimento, foi utilizado um retalho do músculo grande dorsal, com pedículo dorso-caudal, para o reparo de defeitos diafragmáticos iatrogênicos em 18 cães adultos. O acesso ao diafragma foi através de toracotomia no décimo espaço intercostal, sendo criado um defeito diafragmático, onde o retalho foi suturado. $O$ retalho muscular pediculado cobriu um terco do hemidiafragma direito. Os animais tiveram boa recuperação pós-cirúrgica, sem sinais de comprometimento respiratório. Foram feitas necropsias aos 15,30 e 60 dias do pósoperatório. $O$ local submetido ao implante apresentou algumas aderências com vísceras adjacentes e consolidação da sutura macro e microscopicamente. Conclui-se que o retalho pediculado do grande dorsal pode ser útil para o reparo de grandes defeitos diafragmáticos em cães.

Palavras-chave: cirurgia, retalho muscular pediculado, hérnia diafragmática, cão.

\section{SUMMARY}

In this study, the Latissimus dorsi muscular flap was experimentally used for the repair of iatrogenic diaphragmatic defects in 18 adult dogs. The flap was elevated maintaining its dorso-caudal attachment. The diaphragm was accessed through a right thoracotomy in the tenth intercostal space, a defect in the diaphragm was created and the flap was sutured in the margins of the defect. The flap was capable of covering one third of the right hemidiaphragm. All the animals had good post-surgery recoveries. Respiratory function was not compromised. Necropsies were taken at 15, 30 and 60 days post-surgery. Macro and microscopically, the muscle showed few adhesions with adjacent viscera and excellent healing. The Latissimus dorsi muscular flap can be useful for reconstruction of large diaphragmatic defects in dogs.
Key words: surgery, muscular flap, diaphragmatic hernia, dog.

\section{INTRODUÇÃO}

Em geral, o diafragma pode ser reparado cirurgicamente com facilidade (HELPHREY, 1982; RAISER, 1994; HOSGOOD, 1996). Grandes defeitos, entretanto, nem sempre podem ser aproximados por sutura direta, exigindo o uso de enxertos autógenos ou sintéticos (BIANCHI $\boldsymbol{e t}$ al., 1983; DALECK et al., 1988; LALLY et al., 1993). Hérnias crônicas podem não ser facilmente reduzidas quando há contração do diafragma ou aderências com vísceras (HELPHREY, 1982; DALECK et al., 1988; HOSGOOD, 1996).

Vários materiais foram testados para o reparo de grandes defeitos diafragmáticos. Segundo LALLY $\boldsymbol{e t}$ al. (1993), WALLACE \& RODEN (1995) e RAMADWAR $\boldsymbol{e t}$ al. (1997), malhas absorvíveis foram substituídas por uma membrana fibrosa fina que se rompeu, enquanto materiais sintéticos não absorvíveis provocaram deformidade da caixa torácica com o crescimento corporal, necessitando de posterior substituição por retalhos musculares pediculados.

Peritônio bovino conservado em glicerina (DALECK $\boldsymbol{e t} \boldsymbol{a l} ., 1988$ ) e pericárdio canino tratado com compostos poliepoxi (MATSUMOTO et al.,

\footnotetext{
${ }^{1}$ Médico Veterinário, Mestre, Hospital de Clínicas Veterinárias, Universidade Federal do Rio Grande do Sul, 91540-000, Porto Alegre, RS. E-mail: luoliv@myway.com.br. Autor para correspondência.

${ }^{2}$ Médico Veterinário, PhD., Professor Adjunto, Departamento de Clínica de Pequenos Animais, Universidade Federal de Santa Maria (UFSM).

${ }^{3}$ Médico Veterinário, PhD., Professor Titular, Departamento de Patologia, UFSM.

${ }^{4}$ Médico Veterinário, Aluno de Pós-graduação em Medicina Veterinária, UFSM. 
1996) foram utilizados experimentalmente na substituição diafragmática em cães. Foi verificada a incorporação do implante pelo diafragma e ausência de anormalidades no pós-operatório.

Retalho pediculado do músculo transverso abdominal com o peritônio, em cães com hérnias diafragmáticas crônicas, foi estudado por HELPHREY (1982). BIANCHI et al. (1983) recomendaram o uso do retalho pediculado do músculo grande dorsal em agenesia de hemidiafragma em recém-nascidos e no reparo de hérnias diafragmáticas recorrentes. Para reconstrução em massa da parede torácica e diafragma, EDINGTON et al. (1989) utilizaram retalho pediculado do grande dorsal e omento, criando um hemidiafragma funcional num homem adulto. Um retalho livre do grande dorsal foi usado experimentalmente por BARREIROS et al. (1996) como enxerto autógeno para reparar grandes hérnias diafragmáticas em cães.

Os retalhos musculares pediculados fornecem um suporte estrutural para grandes perdas teciduais, além de uma fonte de vascularização às áreas para as quais são transplantados (EDINGTON et al., 1989; PHILIBERT \& FOWLER, 1996). O retalho pediculado deve possuir um bom aporte vascular, um fácil acesso cirúrgico e deve provocar a menor morbidade possível no sítio doador (NICOLL et al., 1996).

O grande dorsal possui duas fontes de suprimento sangüíneo: a artéria toracodorsal, na sua inserção, que é o pedículo dominante, e o pedículo secundário dorso-caudal, que compreende as artérias intercostais (PAVLETIC $\boldsymbol{e t} \boldsymbol{a l}$., 1987; NICOLL $\boldsymbol{e}$ al., 1996; PHILIBERT \& FOWLER, 1996).

O retalho pediculado do grande dorsal tem apresentado grande versatilidade em cirurgias reconstrutivas de pequenos animais. Este trabalho tem como objetivo avaliar a viabilidade do retalho pediculado do grande dorsal, mantendo-se seu pedículo secundário, para o reparo de defeito diafragmático iatrogênico em cães, através de toracotomia lateral intercostal.

\section{MATERIAL E MÉTODO}

Foram utilizados dezoito cães, sem raça definida, adultos, pesando entre 10 e $20 \mathrm{~kg}$, provenientes do Biotério Central da Universidade Federal de Santa Maria. Durante o período experimental, os animais foram alojados no canil do Laboratório de Cirurgia Experimental da mesma Universidade.

Ao iniciar o experimento, os cães foram identificados e vermifugados com associação de praziquantel $(5 \mathrm{mg} / \mathrm{kg})$ e pamoato de pirantel $^{\mathrm{a}}$ (14mg/kg), via oral. Após, passaram por um período de adaptação ambiental de quinze dias e foram alimentados com ração comercial e água à vontade, estando em boas condições clínicas no início do experimento. Os cães foram separados aleatoriamente em três grupos experimentais, I, II e III, cada um contendo 6 deles.

Os animais foram submetidos a jejum sólido por 12 horas no período pré-operatório. Imediatamente antes da cirurgia, foi feita tricotomia da parede tóraco-abdominal direita. Após, foram prémedicados com acepromazina ${ }^{\mathrm{b}}(0,1 \mathrm{mg} / \mathrm{kg})$, submetidos à indução anestésica com tiopental sódico ${ }^{\mathrm{c}}$ $(12,5 \mathrm{mg} / \mathrm{kg})$ e sofreram intubação orotraqueal. A manutenção anestésica foi realizada com circuito semifechado com halotano ${ }^{\mathrm{d}}$ em oxigênio e ventilação controlada ${ }^{\mathrm{e}}$. Os animais receberam, durante a cirurgia, analgesia com fentanil ${ }^{\mathrm{f}}(5 \mathrm{mcg} / \mathrm{kg})$, antibiótico profilático à base de ampicilina ${ }^{\mathrm{g}}(22 \mathrm{mg} / \mathrm{kg})$ e fluidoterapia com ringer lactato $(10 \mathrm{ml} / \mathrm{kg} / \mathrm{h})$ intravenoso.

Após a anti-sepsia, foi feita incisão de pele ao longo do décimo espaço intercostal direito. O grande dorsal foi localizado, divulsionado cranialmente e incisado a, aproximadamente, $3 \mathrm{~cm}$ da sua inserção, mantendo-se a irrigação costal. O retalho foi constantemente irrigado com solução fisiológica durante a cirurgia. Após obtenção do retalho pediculado, foi realizada a toracotomia no mesmo espaço intercostal.

Foram colocados pontos de reparo em cada vértice de um segmento de $6 \times 10 \mathrm{~cm}$ do diafragma, que foi excisado, incluindo parte da porção costal direita e do centro tendíneo. O retalho pediculado foi deslocado para a cavidade torácica, pelo mesmo espaço intercostal da toracotomia, e suturado ao diafragma com fio de seda $2-0^{\mathrm{h}}$, através de pontos de Wolff com sobreposição de bordas.

Para a toracorrafia, foram colocados três pontos isolados para aproximação das costelas anterior e posterior à incisão. $\mathrm{O}$ retalho pediculado foi suturado por pontos isolados simples entre os músculos intercostais, cuidando para não apertar essa sutura. O espaço morto do sítio doador do retalho foi reduzido por "walking suture" com fio mononáilon 3-0 $0^{\mathrm{i}}$. Foi feita sutura contínua simples do tecido subcutâneo e dermorrafia com pontos isolados simples com o mesmo fio.

A pressão negativa intratorácica foi restabelecida por insuflação forçada do pulmão durante o último ponto de vedação da cavidade, além de aspiração com agulha, seringa e torneira de 3 vias após a cirurgia. Para analgesia pós-cirúrgica, foi aplicada morfina via epidural ${ }^{\mathrm{j}}(0,1 \mathrm{mg} / \mathrm{kg})$, imediatamente após a cirurgia, e flunixin-meglumine ${ }^{1}(1,1 \mathrm{mg} / \mathrm{kg})$, 
via intramuscular, nos três primeiros dias do pósoperatório.

A ferida cirúrgica foi higienizada diariamente com solução fisiológica até a retirada dos pontos, após 7 dias. Os animais foram avaliados clinicamente através da freqüência cardíaca e respiratória e volume corrente até 8 horas após a cirurgia e antes das necropsias.

Os animais do grupo I foram sacrificados com 15 dias de pós-operatório, os do grupo II com 30 e, os do grupo III, com 60 dias, sendo observado, macroscopicamente, a cicatrização do retalho pediculado com o diafragma e a presença de alterações no diafragma ou nas cavidades torácica e abdominal. Biópsias, compreendendo parte do diafragma e do retalho implantado, foram coletadas e fixadas em formol tamponado a $10 \%$ para posterior exame histopatológico em microscopia óptica.

\section{RESULTADOS E DISCUSSÃO}

O procedimento cirúrgico foi de fácil execução, sendo o retalho pediculado do grande dorsal dissecado com facilidade e rapidez em todos os cães, conforme já fora relatado por GREGORY et al. (1988). Os estudos de PAVLETIC et al. (1987), GREGORY et al. (1988) e PHILIBERT \& FOWLER (1996) sobre a anatomia e suprimento vascular do grande dorsal, auxiliaram esse procedimento. O retalho pediculado foi divulsionado de maneira atraumática, buscando-se preservar sua rede vascular. As artérias intercostais, no oitavo e nono espaço intercostal, causaram sangramento, quando seccionadas, e precisaram ser ligadas. As ligaduras não prejudicaram a perfusão sangüínea para o músculo, a julgar pela aparência e temperatura normais apresentadas até o final da cirurgia. A ligadura de parte do aporte vascular, diminuindo a base do retalho, não prejudicou sua viabilidade e aumentou sua versatilidade, estando de acordo com BIANCHI $\boldsymbol{e t}$ al. (1983), GREGORY et al. (1988) e WALLACE \& RODEN (1995). Já segundo PAVLETIC $\boldsymbol{e t}$ al. (1987) e PHILIBERT \& FOWLER (1996), essas ligaduras inviabilizariam o retalho pediculado do grande dorsal.

As dimensões do retalho pediculado do grande dorsal se mostraram adequadas para o reparo do diafragma, sendo possível substituir até uma terça parte do mesmo. A toracotomia no décimo espaço intercostal permitiu bom aproveitamento do grande dorsal, produzindo um retalho pediculado extenso. De acordo com NICOLL et al. (1996), o tamanho do retalho pediculado do grande dorsal, geralmente, excede o tamanho requerido para a maioria das situações clínicas, permitindo grande flexibilidade quanto ao seu uso.
O acesso cirúrgico utilizado permitiu a visualização das cavidades torácica e abdominal, a manipulação das vísceras e um acesso adequado à porção costal do diafragma. Este acesso também foi usado por DALECK $\boldsymbol{e t}$ al. (1988), RAISER (1994), WALLACE \& RODEN (1995) e BARREIROS $\boldsymbol{e t}$ al. (1996). Ao contrário, BIANCHI et al. (1983) e HOSGOOD (1996) recomendaram a via abdominal para abordar o diafragma.

A toracotomia pelo décimo espaço intercostal, por ser uma abordagem mais caudal da cavidade torácica, dificultou o acesso à cúpula diafragmática, porém não impediu a correção cirúrgica. Concordando com WALLACE \& RODEN (1995), a utilização desse espaço intercostal permitiu todos os procedimentos cirúrgicos por uma única incisão. BIANCHI et al. (1983) descreveram o reparo de hérnia diafragmática com uso do retalho pediculado do grande dorsal em crianças, através de três diferentes incisões, uma para divulsão do retalho pediculado, outra para toracotomia com ressecção de uma costela, e uma terceira incisão para laparotomia e sutura do retalho pediculado ao diafragma.

A sutura do retalho pediculado ao diafragma por pontos de Wolff, com sobreposição de bordas, com seda 2-0, demonstrou boa coaptação sem tensão no retalho pediculado. A seda foi escolhida, neste experimento, por ser um material não absorvível de baixo custo. BOUDRIEAU (1996) e HOSGOOD (1996) também recomendaram materiais não absorvíveis, pela sua força tênsil prolongada.

A toracorrafia com manutenção do pedículo do grande dorsal entre os músculos intercostais, sem ressecção de costela, não apresentou isquemia por compressão do pedículo. Esse risco foi citado por BIANCHI et al. (1983) e WALLACE \& RODEN (1995).

O uso combinado dos métodos de insuflação pulmonar forçada e aspiração foi eficiente para restabelecer a pressão negativa intratorácica. RAISER (1994) utilizou a insuflação pulmonar com sucesso após herniorrafias diafragmáticas, sendo que alguns animais necessitaram toracocentese complementar. HOSGOOD (1996) sugeriu o uso de dreno de toracostomia para facilitar o acesso a complicações como pneumo ou hemotórax pós-cirúrgico. BOUDRIEAU (1996) igualmente recomendou dreno de toracostomia e citou o risco de laceração pulmonar nas aspirações através de agulha.

Nas primeiras horas após a cirurgia, os valores de volume corrente diminuíram e os de frequiência respiratória estiveram elevados, provavelmente devido à dor. Após 24 horas da cirurgia, os cães apresentavam-se bem dispostos e com apetite normal, sem sinais de dor, nem alterações respiratórias. 
A elevação de um retalho pediculado com grandes dimensões cria um espaço morto extenso no sítio doador. A "walking suture", utilizada no local, mostrou-se adequada para a redução desse espaço. Três cães desenvolveram seromas no sítio doador, que foram drenados por aspiração com seringa e agulha, sendo uma única drenagem suficiente. Os animais não mostraram alterações clínicas devido aos seromas. PAVLETIC et al. (1987) relataram que seromas, deiscência e necrose, resultado de circulação insuficiente, não afetaram a sobrevivência de retalhos pediculados miocutâneos do grande dorsal em cães. Estudando retalhos pediculados do grande dorsal em gatos, NICOLL et al. (1996) notaram a formação de seromas que cederam após tratamento, também sem complicações.

No local doador do retalho pediculado, não foram evidenciadas deformidades físicas ou funcionais, como também foi relatado por BIANCHI et al. (1983) e EDINGTON et al. (1989).

Nas necropsias, foi confirmada a integridade do retalho muscular pediculado e sua cicatrização com o diafragma (Figura 1). Foram observadas aderências do retalho pediculado com o fígado em todos os cães. Quinze cães tiveram aderência do diafragma com o lobo caudal do pulmão direito, quatro apresentaram aderência com o omento (aos 60 dias de observação) e, um, aderência com o estômago (30 dias de observação). A ausência de uma superfície serosa, o uso de fio reativo e não absorvível, além da extensão do retalho pediculado, que exige bastante manipulação cirúrgica, provavelmente foram a causa dessas aderências. Entretanto, elas não levaram a alterações clínicas. A formação de aderências poderia ser minimizada com o uso de fio menos reativo que a seda e com a utilização de técnica cirúrgica cuidadosa. HELPHREY (1982) observou que a presença de uma superfície serosa no implante evita aderências. MATSUMOTO et al.

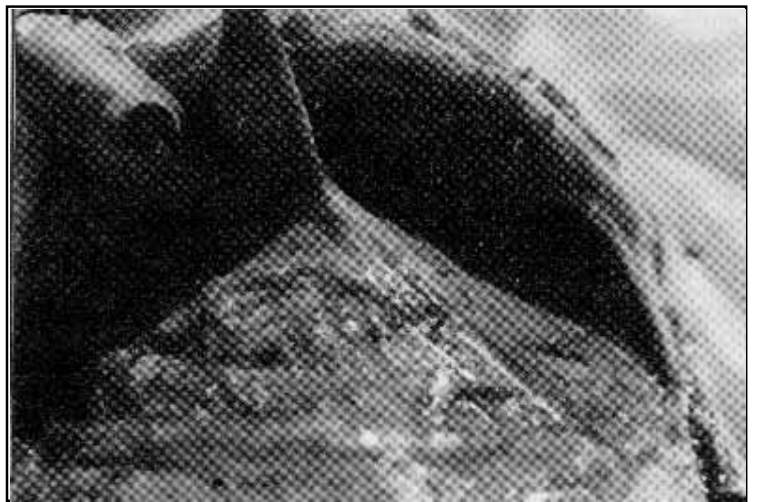

Figura 1 - Aspecto macroscópico da anastomose entre o grande dorsal e o diafragma, num cão, 30 dias após a cirurgia.
(1996) atribuíram as aderências ao fio de seda utilizado na sutura.

Nenhum animal apresentou eventrações, o que ocorreu em experimentos de LALLY et al. (1993) e RAMADWAR et $\boldsymbol{a l}$. (1997), ao utilizarem malhas sintéticas absorvíveis em hérnias diafragmáticas. As malhas foram substituídas por membranas fibrosas finas que romperam, possivelmente em função da tensão criada pela constante movimentação do diafragma ou pela absorção precoce das malhas, que resultaria na proliferação inadequada de fibroblastos.

Nos exames microscópicos dos animais avaliados aos 15 dias após a cirurgia, foram observadas discreta hemorragia na serosa e no tecido conjuntivo, espessamento dos septos conjuntivos e algumas células inflamatórias nos fascículos e no tecido de granulação. Em outros fascículos, houve proliferação de tecido fibroso. Aos 30 dias após a cirurgia, foi observado tecido de granulação maduro unindo os músculos, com infiltração gordurosa multifocal e infiltrado inflamatório focal misto. Em alguns fascículos, foi observada invasão moderada de tecido fibroso. Nos exames, aos 60 dias após a cirurgia, a maioria dos fascículos apresentou invasão abundante de colágeno.

Analisando o resultados dos exames microscópicos, foram evidenciadas uma reação inflamatória e crescimento de tecido conjuntivo, caracterizando a resposta cicatricial do diafragma com o enxerto, apresentando uma boa consolidação da sutura. Reações semelhantes foram observadas por DALECK $\boldsymbol{e t}$ al.(1988), BARREIROS $\boldsymbol{e t}$ al. (1996) e MATSUMOTO et al. (1996). Foi observada uma resposta granulomatosa ao fio de seda, o que caracteriza uma reação do tipo corpo estranho, já que esse fio não é absorvível e se caracteriza por provocar uma reação inflamatória acentuada. O uso de um fio de sutura inerte poderia minimizar essa reação.

O retalho pediculado do músculo grande dorsal, mantendo-se seu pedículo secundário dorsocaudal, é uma opção para a correção de grandes defeitos diafragmáticos em cães, mediante acesso pela via torácica lateral intercostal.

\section{FONTES DE AQUISIÇÃO}

\footnotetext{
a - Drontal: Bayer, São Paulo, SP.

b - Acepram: Univet, São Paulo, SP.

- Thionembutal: Abbott, São Paulo, SP.

d - Halotano: Hoechst, São Paulo, SP.

- Ventilador Takaoka 671 e Circuito Takaoka série KT13: K Takaoka, São Paulo, SP.

f - Fentanil: Janssen, São José dos Campos, SP.

g - Ampicilina: Univet, São Paulo, SP.

h - Seda 2-0: Ethicon - Johnson \& Johnson, São José dos Campos, SP.
} 
i - Mononylon 3-0: Ethicon - Johnson \& Johnson, São José dos Campos, SP.

j - Dimorf-SP: Innovatec - Cristália, Itapira, SP

' - Banamine: Schering-Plough, Rio de Janeiro, RJ.

\section{REFERÊNCIAS BIBLIOGRÁFICAS}

BARREIROS, L.J., RODASKI, S., SUSKO, I., et al. Uso experimental do músculo grande dorsal autólogo na reparação dos grandes defeitos diafragmáticos no cão. Revista do Setor de Ciências Agrárias, v.15, n.1, p.141-150, 1996.

BIANCHI, A., DOIG, C.M., COHEN, S.J. The reverse Latissimus dorsi flap for congenital diaphragmatic hernia repair. Journal of Pediatric Surgery, v.18, n.5, p.560-563, 1983.

BOUDRIEAU, R.J. Hérnia diafragmática traumática. In: BOJRAB, M.J. (Editor). T écnicas atuais em cirurgia de pequenos animais. 3.ed. São Paulo : Roca, 1996. Cap.17, p.293-297.

DALECK, C.R., DALECK, C.L.M., ALESSI, A.C., et al. Substituição de um retalho diafragmático de cão por peritônio bovino conservado em glicerina: estudo experimental. Ars Veterinária, v.4, n.1, p.53-61, 1988.

EDINGTON, H.D., EVANS, S. SINDELAR, W.F. Reconstruction of a functional hemidiaphragm with use of omentum and Latissimus dorsi flaps. Surgery, v.105, n.3, p.442-445, 1989.

GREGORY, C.R., GOURLEY, I.M., KOBLIK, P.D., et al. Experimental definition of Latissimus dorsi, gracilis and Rectus abdominis musculocutaneous flaps in the dog. American Journal of Veterinary Research, v.49, n.6, p.878-884, 1988.

HELPHREY, M.L. Abdominal flap graft for repair of chronic diaphragmatic hernia in the dog. Journal of the American Veterinary Medical Association, v.181, n.8, p.791-793, 1982.
HOSGOOD, G. Diagnosis and management of diseases of the diaphragm. Waltham Focus International, v.6, n.3, p.2-9, 1996.

LALLY, K.P., CHEU, H.W., VAZQUEZ, W.D. Prosthetic diaphragm reconstruction in the growing animal. Journal of Pediatric Surgery, v.28, n.1, p.45-47, 1993.

MATSUMOTO, H., OGUCHI, Y., MIYAKE, Y., et al. The use pf epoxy patch grafts for the repair of experimentally-created diaphragmatic defects in dogs. Journal of Veterinary Medical Sciences, v.58, n.7, p.685-687, 1996.

NICOLL, S.A., FOWLER, J.D., REMEDIOS, A.M., $\boldsymbol{e t}$ al Development of a free Latissimus dorsi muscle flap in cats. Veterinary Surgery, v.25, n.1, p.40-48, 1996.

PAVletiC, M.M., KOSTOLICH, M., KOBLIK, P., et al. A comparison of the Cutaneous trunci myocutaneous flap and latissimus dorsi myocutaneous flap in the dog. Veterinary Surgery, v.16, n.4, p.283-293, 1987.

PHILIBERT, D., FOWLER, J.D. Use of muscle flaps in reconstructive surgery. Compendium on Continuing Education for the Practicing Veterinarian, v.18, n.4, p.395-404, 1996.

RAISER, A.G. Herniorrafia diafragmática em cães e gatos. Relato de 22 casos e proposição de técnica para corrigir rupturas freno-costais. Brazilian Journal of Veterinary Research and Animal Science, v.31, n.3/4, p.245-251, 1994.

RAMADWAR, R.H., CARACHI, R., YOUNG, D.G. Collagencoated vicryl mesh is not a suitable material for repair of diaphragmatic defects. Journal of Pediatric Surgery, v.32, n.12, p.1708-1710, 1997.

WALLACE, C.A., RODEN, J.S. Reverse, innervated Latissimus dorsi flap reconstruction of congenital diaphragmatic absence. Plastic and Reconstructive Surgery, v.96, n.4, p.761-769, 1995.

Ciência Rural, v. 30, n. 6, 2000. 